\title{
Sliced Ridgelet Transform for Image Denoising
}

\author{
V.Krishna Naik 1, Prof.Dr.G.Manoj Someswar 2, R.B. Dayananda 3 \\ Asst. Professor, Department of Electronics \& Communications Engineering, Axsum University, Ethiopia, \\ Africa. \\ Prof.Dr.G.Manoj Someswar, Principal \& Professor, Department of Computer Science \& Enginering, Anwar-ul- \\ uloom College of Engineering \&Technology, Yennepally, RR District, Vikarabad-501101, A.P., India. \\ Dayananda R. B., Asst. Professor, Department of Computer Science \& Engineering, Raja Reddy Institute of \\ Technology, Chikkabanavara, Bangalore, Karnataka, India.
}

\begin{abstract}
Image denoising based on ridgelet transforms gives better result in image denoising than standard wavelet transforms. In this research work, the researcher introduces a new approach for image denoising that is based on ridgelets computed in a localized manner and that is computationally less intensive than curvelets, but similar donising performance. The projection of image at a certain angle is computed at a certain angle, but only on a defined slice of the noisy image. After that, ridgelet transform of each slice is computed, to produce the ridgelet coefficients for an image. The denoising operation corresponds to a simple thresholding of these ridgelet coefficients.

The new method for image denoising technique is based on two operations: one is the redundant directional wavelet transform based on the radon transform, and thresholding of the ridgelet coefficient.

The image denoising algorithm with the ridgelet transform can be described by the following operations. First , add the noise to an image and than projection(radon transform) is computed at a certain angle of the noise image. After that, the ridgelet transform of this projection of the noise image is computed and the noise component is reduced by simple thresholding of the ridgelet coefficient. Then, the inverse ridgelet transform is computed to get back the denoised version of that projection of slice at the same angle. Although the shape of the reconstructed object can be seen, the reconstructed image is heavily blurred. To counteract this effect, a high pass filter is applied to the sinogram data in the frequency domain. This is achieved by applying a 1-D DFT to the sinogram data for each angle, multiply by the filter, and then using the inverse DFT to reconstruct the data. The simplest form of high pass filter is a ramp. Applying the ramp filter significantly improves the quality of the reconstructed image. However, because the ramp filter emphasises high frequency components of the image, it can cause unwanted noise. To counteract this, several other high-pass filters are commonly used. In this project we are using Adaptive Filtering. The wiener 2 function applies a Wiener filter (a type of linear filter) to an image adaptively, tailoring itself to the local image variance. Where the variance is large, wiener2 performs little smoothing. Where the variance is small, wiener 2 performs more smoothing. This approach often produces better results than linear filtering. The adaptive filter is more selective than a comparable linear filter, preserving edges and other high-frequency parts of an image. In addition, there are no design tasks; the wiener 2 function handles all preliminary computations and implements the filter for an input image. wiener 2.

However, does require more computation time than linear filtering. Wiener 2 works best when the noise is constant-power ("white") additive noise, such as Gaussian noise.
\end{abstract}

\section{Introduction:}

Image denoising is one of the most popular research fields in image processing due to fact that it is extremely difficult to form a general global denoising scheme effective for all types of noise as well as all types images. A common problem in image denoising is the blurring of the prominent edges in the image which can cause discrepancies when the denoising operation is combined with other operations such as image edge detection and segmentation. Notewothy schemes for image denoising based on transforms such as wavelets, curvelets, exploit redundancy and thresholding to remove the noise without blurring the edges. The important characteristic of the denoising technique introduced in this project is that it can reduce considerably the noise without destroying the edges of the objects in the image. That is, the noise is well attenuated but the edge information is preserved. The new method for image denoising technique is based on two operations: one is the redundant directional wavelet transform based on the ridgelet transform, and thresholding of the ridgelet coefficient.

The image denoising algorithm with the ridgelet transform can be described by the following operations. First, add the noise to an image and than projection(ridgelet transform) is computed at a certain angle, but only on a defined silce of the noise image. After that, the ridgelet transform of this projection of the noise image is computed and the noise component is reduced by simple thresholding of the ridgelet coefficient. 
Then, the inverse ridgelet transform is computed to get back the denoised version of that projection of the defined slice at the same angle. Although the shape of the reconstructed object can be seen, the reconstructed image is heavily blurred. To counteract this effect, a high pass filter is applied to the sinogram data in the frequency domain. The wiener2 function applies a Wiener filter (a type of linear filter) to an image adaptively, tailoring itself to the local image variance. Where the variance is large, wiener2 performs little smoothing. Where the variance is small, wiener2 performs more smoothing. This approach often produces better results than linear filtering.

The adaptive filter is more selective than a comparable linear filter, preserving edges and other highfrequency parts of an image. In addition, there are no design tasks; the wiener2 function handles all preliminary computations and implements the filter for an input image. wiener2, however, does require more computation time than linear filtering. wiener2 works best when the noise is constant-power ("white") additive noise, such as Gaussian noise.

\section{Radon Transform:}

\section{Experiment Design And Setup}

The Radon transform of an image is the sum of the Radon transforms of each individual pixel.The algorithm first divides pixels in the image into four subpixels and projects each subpixel separately.

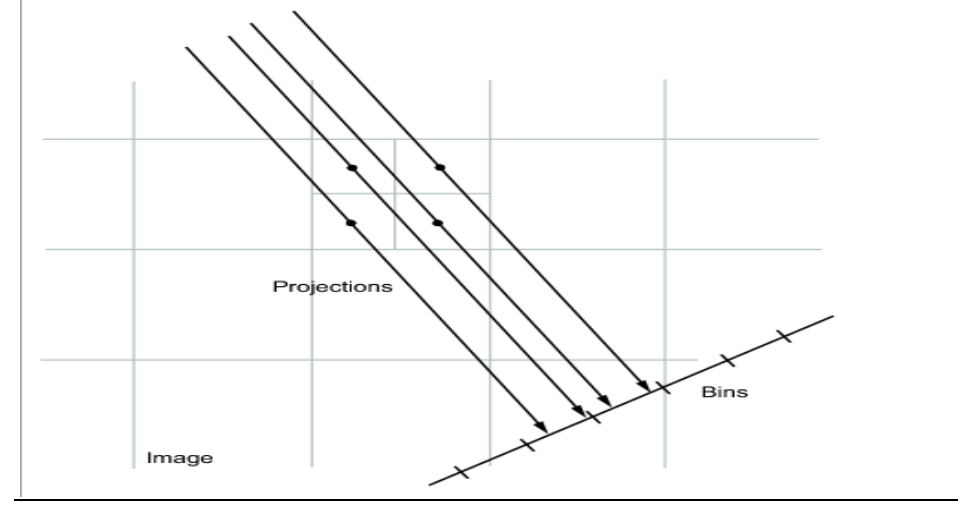

Figure 1

Each subpixel's contribution is proportionally split into the two nearest bins, according to the distance between the projected location and the bin centers. If the subpixel projection hits the center point of a bin, the bin on the axes gets the full value of the subpixel, or one-fourth the value of the pixel. If the subpixel projection hits the border between two bins, the subpixel value is split evenly between the bins.

\section{Radon Transformation Definition}

The radon function computes projections of an image matrix along specified directions. A projection of a two-dimensional function $\mathrm{f}(\mathrm{x}, \mathrm{y})$ is a set of line integrals. The radon function computes the line integrals from multiple sources along parallel paths, or beams, in a certain direction. The beams are spaced 1 pixel unit apart. To represent an image, the radon function takes multiple, parallel-beam projections of the image from different angles by rotating the source around the center of the image. The following figure shows a single projection at a specified rotation angle.

\section{Parallel-Beam Projection at Rotation Angle Theta}

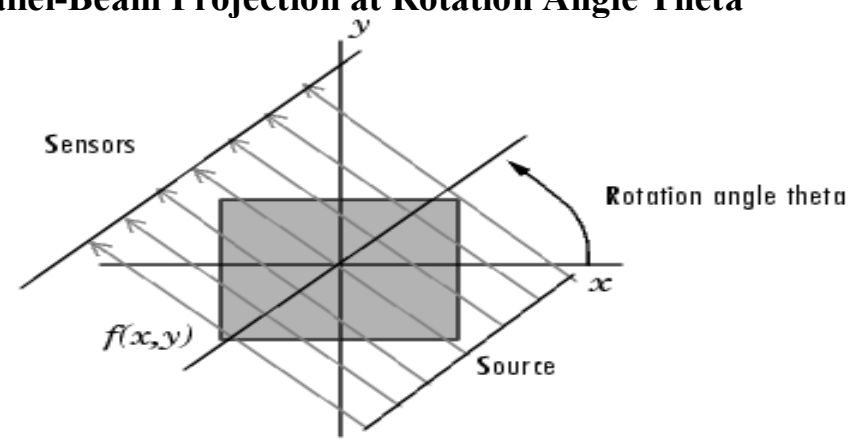

Figure 2 
For example, the line integral of $f(x, y)$ in the vertical direction is the projection of $f(x, y)$ onto the $x$-axis; the line integral in the horizontal direction is the projection of $f(x, y)$ onto the $y$-axis. The following figure shows horizontal and vertical projections for a simple two-dimensional function.
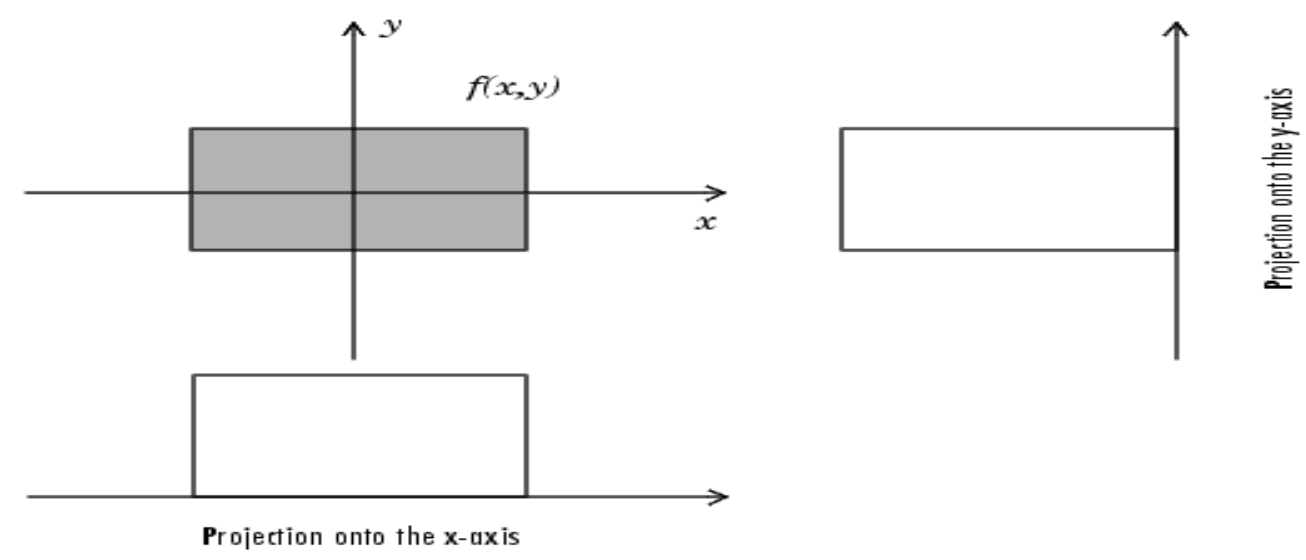

Figure 3

Projections can be computed along any angle $\boldsymbol{\theta}$. In general, the Radon transform of $f(x, y)$ is the line integral of $f$ parallel to the $y^{\prime}$-axis

$R_{\theta}\left(x^{\prime}\right)=\int_{-\infty}^{\infty} f\left(x^{\prime} \cos \theta-y^{\prime} \sin \theta, x^{\prime} \sin \theta+y^{\prime} \cos \theta\right) d y^{\prime}$

Where

$\left[\begin{array}{l}x^{\prime} \\ y^{\prime}\end{array}\right]=\left[\begin{array}{rr}\cos \theta & \sin \theta \\ -\sin \theta & \cos \theta\end{array}\right]\left[\begin{array}{l}x \\ y\end{array}\right]$

The following figure illustrates the geometry of the Radon transform.

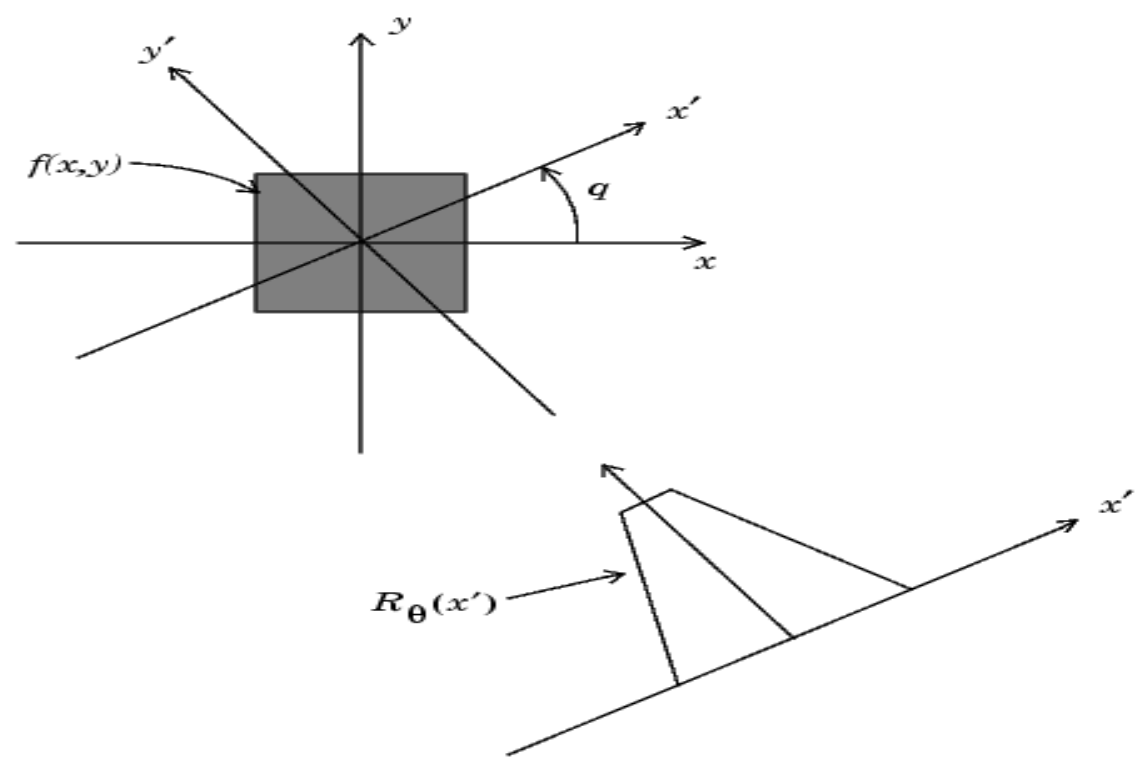

Figure 4

\section{Geometry of the Radon Transform}

This command computes the Radon transform of I for the angles specified in the vector theta.

$[\mathrm{R}, \mathrm{xp}]=\operatorname{radon}(\mathrm{I}$, theta $)$;

The columns of $\mathrm{R}$ contain the Radon transform for each angle in theta. The vector xp contains the corresponding coordinates along the $x^{\prime}$-axis. The center pixel of I is defined to be floor((size $\left.\left.(\mathrm{I})+1\right) / 2\right)$; this is the pixel on the $x^{\prime}$-axis corresponding to $x^{\prime}=0$. 
The Radon transform of an image represented by the function $f(x, y)$ can be defined as a series of line integrals through $\mathrm{f}(\mathrm{x}, \mathrm{y})$ at different offsets from the origin. It can be defined mathematically as

$$
R(p, \tau)=\int_{-\infty}^{\infty} f(x, p x+\tau) d x
$$

where $\mathrm{p}$ and tau are the slope and intercepts of the line.

A more directly applicable form of the transform can be defined by using a delta function:

$$
R(r, \theta)=\int_{-\infty}^{\infty} \int_{-\infty}^{\infty} f(x, y) \delta(x \cos \theta+y \sin \theta-r) d x d y
$$

where theta is the angle of the line, and $r$ is the perpendicular offset of the line.

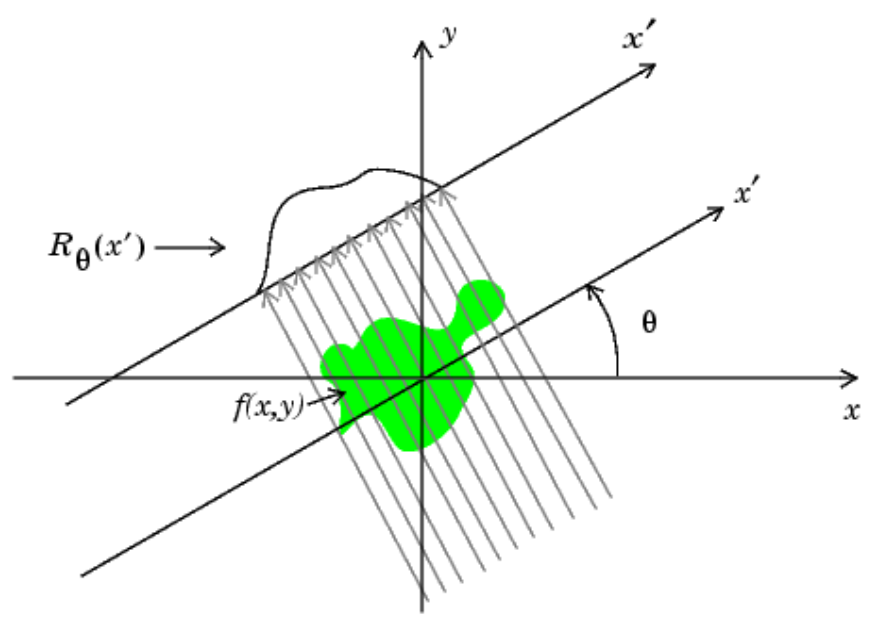

Figure 5

\section{Radon Transform}

The acquisition of data in medical imaging techniques such as MRI, CT and PET scanners involves a similar method of projecting a beam through an object, and the data is in a similar form to that described in the second equation above. The plot of the Radon transform, or scanner data, is referred to as a sinogram due to its characteristic sinusoid shape. Figure 2 shows a simple non-homogeneous shape and the sinogram created by taking the Radon transform at intervals of one degree from 0 to 180 degrees.

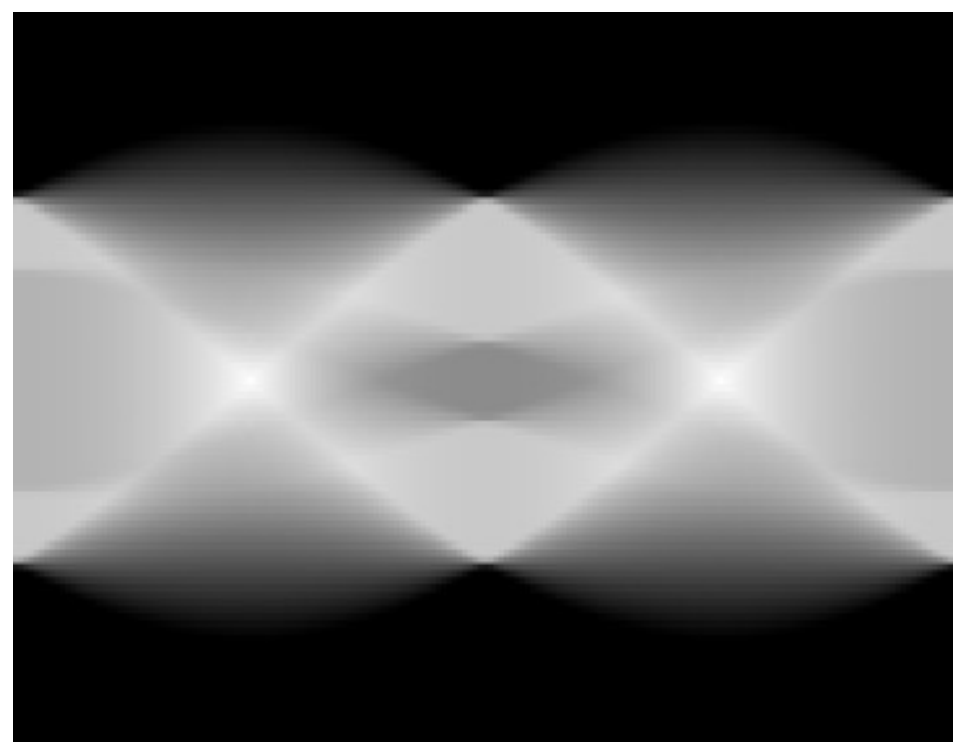

Figure 6 


\section{The sinogram produced by applying the Radon Transform.}

\section{INVERS RADON TRANSFORM USING FILTERED BACK PROJECTION:}

To reconstruct the image from the sinogram, the inverse Radon transform is applied to the image. There are several techniques by which the inverse transform can be calculated but the most common is Filtered Back Projection.

The filtered back projection algorithm is split into two phases, filtration and projection.

\section{PROJECTION:}

The projection phase is very similar to the Radon transform described above, and shown in Figure 1 except now the line integrals are projected back onto the plane at their respective angles. The projection phase of the Filtered Back Projection, using the data format described above:

$$
f(x, y)=\int_{0}^{\pi} f^{\prime}(x \cos \theta+y \sin \theta, \theta)
$$

where $\mathrm{f}^{\prime}$ is the filtered data.

A common discrete approximation of this is:

$$
f\left(x_{m}, y_{n}\right) \approx \Delta \theta \sum_{t=0}^{T-1} f^{\prime}\left(x_{m} \cos \theta_{t}+y_{n} \sin \theta_{t}, \theta_{t}\right)
$$

This equation can be used to determine the pixel values at a given point. The exact values depend on the chosen interpolation method, e.g. nearest-neighbour, linear interpolation etc. As more projections are added, the quality of the reconstruction will increase. This is clearly not enough to accurately reconstruct the image, but the individual projections can be seen. the result of the back projection algorithm applied as described and using all available data. Although the shape of the reconstructed object can be seen, the reconstructed image is heavily blurred. To counteract this effect, a high pass filter is applied to the sinogram data in the frequency domain. This is achieved by applying a 1-D DFT to the sinogram data for each angle, multiply by the filter, and then using the inverse DFT to reconstruct the data. The simplest form of high pass filter is a ramp.

Applying the ramp filter significantly improves the quality of the reconstructed image. However, because the ramp filter emphasises high frequency components of the image, it can cause unwanted noise. To counteract this, several other high-pass filters are commonly used.

\section{Results \& Discussion}

The wiener2 function applies a Wiener filter (a type of linear filter) to an image adaptively, tailoring itself to the local image variance. Where the variance is large, wiener2 performs little smoothing. Where the variance is small, wiener 2 performs more smoothing.This approach often produces better results than linear filtering. The adaptive filter is more selective than a comparable linear filter, preserving edges and other highfrequency parts of an image. In addition, there are no design tasks; the wiener2 function handles all preliminary computations and implements the filter for an input image. wiener2, however, does require more computation time than linear filtering. wiener2 works best when the noise is constant-power ("white") additive noise, such as Gaussian noise.

\section{References}

[1] A. Averbuch, R. R. Coifman, D. L. Donoho, M. Israeli, and J. Waldén, "Polar FFT, rectopolar FFT, and applications," Stanford Univ., Stanford, CA, Tech. Rep., 2000.

[2] "Monoscale ridgelets for the representation of images with edges,”Dept. Statist., Stanford Univ., Stanford, CA, Tech. Rep., 1999, submitted for publication.

[3] "Curvelets-A surprisingly effective nonadaptive representationfor objects with edges," in Curve and Surface Fitting: Saint-Malo 1999,A. Cohen, C. Rabut, and L. L. Schumaker, Eds. Nashville, TN: Vanderbilt Univ. Press, 1999.

[4] G.Y. Chen, T.D. Bui, A. Krzy zak, Image denoising with neighbour dependency and customized wavelet and threshold, Pattern Recognition 38 (1) (2005) 115-124.

[5] D.L. Donoho, A.G. Flesia, in: J. Stoecker, G.V. Welland (Eds.), Digital ridgelet transform based on true ridge functions, Beyond Wavelets, Academic Press, New York, 2001.

[6] G.Y. Chen, T.D. Bui, A. Krzy zak, Rotation invariant pattern recognition using ridgelet, wavelet cycle-spinning and Fourierfeatures, Pattern Recognition 38 (12) (2005) 2314-2322.

[7] J.L. Starck, E.J. Candes, D.L. Donoho, Astronomical image representation by the curvelet transform, Astron. Astrophys. 2 (2003) 785-800. 